\title{
Big data and smart computing in network systems
}

\author{
Jiming Chen ${ }^{1} \cdot$ Kaoru Ota ${ }^{2} \cdot$ Lu Wang $^{3} \cdot$ Jianping $\mathrm{He}^{4}$ \\ Published online: 11 July 2019 \\ (C) Springer Science+Business Media, LLC, part of Springer Nature 2019
}

In recent years, big data and smart computing are emerging research fields that have drawn much attention from computer science, communication and control area, information technology as well as from social sciences and other disciplines.

With the increasing deployment of new monitoring and sensing devices, and the advanced measurement infrastructures, a large amount of data is collected in network systems. Network systems have become data-driven, which call for big data, smart computing methods and solutions (e.g., predictive data mining, robust data analytics, artificial intelligence, distributed and high-performance computing, efficient data management, privacy-preserving data publishing, etc.). With the growing volume, speed and types of big data from the network systems, smart computing is imperative to guarantee critical functionalities in network systems, such as real-time widearea situational awareness, dynamic data management, efficiency optimization and control, robust network performance, etc.

The focus of this special issue, "Big data and smart computing in network systems", is on the improvement of network systems operations and applications with emphasis on big data

This article is part of the Topical Collection: Special Issue on Big Data and Smart Computing in Network Systems

Guest Editors: Jiming Chen, Kaoru Ota, Lu Wang, and Jianping He

Jianping $\mathrm{He}$

jphe@sjtu.edu.cn

Jiming Chen

jmchen@ieee.org

Kaoru Ota

ota@csse.muroran-it.ac.jp

Lu Wang

wanglu@szu.edu.cn

1 Zhejiang University, Zhejiang, China

2 Muroran Institute of Technology, Hokkaido, Japan

3 Shenzhen University, Shenzhen, Guangdong Province, China

4 Shanghai Jiao Tong University, Shanghai, China and smart computing technologies. We solicit and publish original research papers on the theories, algorithms, and methodologies that highlight emerging data processing technologies for big data and smart computing. This special issue received 25 submissions and only 13 of them were finally accepted. The accepted papers address a variety of topics, including techniques, models and algorithms for big data in network systems, and networked infrastructure for smart computing, and cloud and grid computing, and data processing approaches for $\mathrm{P} 2 \mathrm{P}$ communication networks, etc. A brief overview of the papers included in the special issue is provided below.

Yu et al. [1] consider the problem that traditional compressive sensing methods are open-loop and the coordinator cannot obtain the recovery quality, which will impact the diagnosis results based on the recovered data. A novel close-loop structure is designed for wireless ECG monitoring, which can maintain the recovery quality and the energy efficiency at a high level. Moreover, an improved Subspace Pursuit recovery method is designed to optimize the recovery quality.

Aguilar et al. [2] consider the agile methods of content distribution, such as general-purpose files, software patches, image files, and other information for the rise in cloud storage and services in enterprise networks. The authors propose a peer selection algorithm in BitTorrent to distribute content in enterprise networks. Peers execute this algorithm with a new special role called Internal Swarm Coordinator, ISC for short.

M. Alshareef et al. [3] use different metrics at various levels to estimate the impact of produced research, including the journal-level impact factor, the number of citations at an article-level and the H-index at an author-level. The authors propose an approach to measure the Article Citation Impact (ACI) that will enable idenGEAtification of the impact of articles at their extended nearby citation network.

Zhang et al. [4] consider the challenge that how to build the trust between the driver and the passenger and achieve the secure billing. They propose a novel secure billing protocol which removes the presence of the online third party by a smart contract on a publicly-verifiable two-party blockchain.

$\mathrm{Li}$ et al. [5] propose a simple and effective method for semisupervised deep learning that improves upon the performance 
of the deep learning model. First, they train a classifier and use its outputs on unlabeled data as pseudo-labels to facilitate the utilization of large-scale unlabeled data. Then, the authors pretrain the deep learning model with the pseudo-labeled data and fine-tune it with the labeled data.

Yuan et al. [6] consider the problem that the existed rangebased 3D target tracking algorithms are generally inaccurate on detecting underwater non-cooperative objects due to the high response time and non-line-of-sight(NLOS) propagation in the underwater acoustic sensor networks (UASNs). A low computational complexity approach namely two-step least square (LS) is proposed to determine the best location by analyzing the distribution of estimated coordinates.

Jiang et al. [7] make detailed analyses of social networks' graph data and state-of-the-art graph computing frameworks, and find that some techniques of the current graph computing systems are overgeneralized and suboptimal, which means they only focus on how to design a graph processing framework on general graphs but miss the optimization of social networks graphs. The authors propose Quick-Squad, a graph computing system on a single server which is specific to the optimization of social networks graph structures.

Lei et al. [8] investigate the problem on modeling the topics emerged in social networks. They propose dynamic topic modeling via a self-aggregation method which can aggregates the observable and unordered short texts as the aggregated document without leveraging an external context. And they exploit word pairs instead of words for each microblog to generate more word co-occurrence patterns.

Zhao et al. [9] focus on improving the computing performance in edge computing, they develop a stochastic load scheduling framework to minimize the communication load under time-varying excess computational resources. They obtain the optimal computation load scheduling algorithm by adopting the augmented Lagrangian method in the offline setting, and they further derive a worst-case performance bound of the online equal task scheduling (ETS) algorithm by using competitive analysis.

Yan et al. [10] consider mining quantitative association rules is one of the most important tasks in data mining, many researches have proved that particle swarm optimization(PSO) algorithm is suitable for quantitative association rule mining (ARM). However, the method becomes inefficient even unavailable on huge datasets. To make up for the deficiency, the authors propose a parallel PSO and design two methods, particle-oriented and data-oriented parallelization, to fit different application scenarios.

Qiao and Yin [11] study the problem on how to improve the quality of service and reduce the cost of content distribution form the perspective of the Internet Content Providers (ICPs). The authors propose a data-driven and the deep learning based CDN recommendation framework for ICPs. They present a three-tier CDN recommendation framework and build a CDN recommendation model based on the deep neural network to improve the efficiency of the recommendation service and satisfy the personalized demand.

Feng et al. [12] study the computation offloading problem under multi-user multi-radio (MUMR) environments. They formulate the problem as a maximization of the total number of beneficial users in consideration of time delay and energy consumption simultaneously. By converting the proposed non-convex mixed integer non-linear programming (MINLP) problem into a bilinear problem, they develop a Branch and Bound algorithm to solve the problem and demonstrate that the proposed method can obtain a near-optimal solution.

Tahmasebi and Khayyambashi [13] consider the novelty of Vehicular Cloud Computing (VCC) due to its efficiently power usage and its significantly reduction on energy consumption, they propose an efficient model that considers expenses and response times which also appropriately utilizes onboard computation capacity for VCC. The proposed model is using VCC in a manner that the onboard computational capability is fully used, so it can provide reasonable response time and makes the model economically beneficial.

Acknowledgments The Guest Editors would like to thank all the authors for their contributions. Their thanks also go to all the anonymous reviewers for the valuable and constructive reviews. In addition, the Guest Editors would like to give our gratitude to Editor-in-Chief of the PPNA, Prof. Xuemin S. Shen, for his generous supporting in organizing this Special Section and the Journal Administrators, Ms. Melissa, for her administrative assistance throughout the development of this special issue.

\section{References}

1. Yu W, Chen C, Liu Z, Yang B, Guan X (2019) DSESP: dual sparsity estimation subspace pursuit for the compressive sensing based close-loop ecg monitoring structure. https://doi.org/10.1007/ s12083-019-00731-5

2. Aguilar A, Lozoya C, Orona LM (2019) A hamming distance and fuzzy logic-based algorithm for P2P content distribution in enterprise networks. https://doi.org/10.1007/s12083-018-0711-8

3. Alshareef AM, Alhamid MF, El Saddik A (2018) Toward citation recommender systems considering the article impact in the extended nearby citation network. https://doi.org/10.1007/s12083-0180687-4

4. Zhang H, Deng E, Zhu H, Cao Z. Smart contract for secure billing in ride-hailing service via blockchain. https://doi.org/10.1007/ s12083-018-0694-5

5. Li Z, Ko BS, Choi H-J (2018) Naive semi-supervised deep learning using pseudo-label. https://doi.org/10.1007/s12083-018-0702-9

6. Yuan Y, Li Y, Liu Z, Chan KY, Zhu S, Guan X (2018) A three dimensional tracking scheme for underwater non-cooperative 
objects in mixed LOS and NLOS environment. https://doi.org/10. 1007/s12083-018-0678-5

7. Jiang X, Li Q, Ma Z, Dong M, Wu J, Guo D. QuickSquad: A new single-machine graph computing framework for detecting fake accounts in large-scale social networks. https://doi.org/10.1007/ s12083-018-0697-2

8. Shi L, Du J, Liang M, Kou F (2018) Dynamic topic modeling via self-aggregation for short text streams. https://doi.org/10.1007/ s12083-018-0692-7

9. Zhao M, Wang W, Wang Y, Zhang Z (2018) Load scheduling for distributed edge computing: A communication-computation tradeoff. https://doi.org/10.1007/s12083-018-0695-4

10. Yan D, Zhao X, Lin R, Bai D (2019) PPQAR: parallel PSO for quantitative association rule mining. https://doi.org/10.1007/ s12083-018-0698-1
11. Qiao B, Yin H. A data-driven and the deep learning based CDN recommendation framework for ICPs. https://doi.org/10.1007/ s12083-018-0673-x

12. Feng G, Lv H, Li B, Wang C, Lv H, Wang H (2018) A near-optimal cloud offloading under multi-user multi-radio environments. https://doi.org/10.1007/s12083-018-0693-6

13. Tahmasebi M, Khayyambashi MR (2018) An efficient model for vehicular cloud computing with prioritizing computing resources. https://doi.org/10.1007/s12083-018-0677-6

Publisher's note Springer Nature remains neutral with regard to jurisdictional claims in published maps and institutional affiliations. 